\title{
El desarrollo de la competencia docente "mirar profesionalmente" la enseñanza- aprendizaje de las matemáticas ${ }^{1}$
}

\section{O desenvolvimento da competência docente de "olhar profissionalmente" 0 ensino-aprendizagem das matemáticas}

\section{The development of the "professional noticing" skill in teaching-learning mathematics}

\author{
Salvador Llinares ${ }^{2}$
}

\begin{abstract}
RESUMEN
Este artículo se inserta en la línea de reflexión en educación matemática centrada en caracterizar y desarrollar la competencia docente "mirar profesionalmente" la enseñanza-aprendizaje de las matemáticas como una componente de la práctica profesional del profesor de matemáticas. Un ejemplo procedente de un curso de formación inicial de profesores nos permite caracterizar su significado. En este trabajo se argumenta que el desarrollo de esta competencia docente se apoya en la relación dialéctica entre el conocimiento de matemáticas para la enseñanza (MKT) y el discurso generado en la resolución de tareas profesionales. Finalmente se describen algunas características de los entornos de aprendizaje en los programas de formación de profesores dirigidos a fomentar el desarrollo de esta relación dialéctica.

Palabras-clave: mirar profesionalmente; formación inicial de profesores; conocimiento de matemáticas para la enseñanza; práctica profesional del profesor de matemáticas.
\end{abstract}

1 Este trabajo ha recibido el apoyo del proyecto I+D+i del Plan Nacional de Investigación del Ministerio de Ciencia e Innovación, España, EDU2011-27288.

2 Universidad de Alicante. Alicante, España. Carretera de San Vicente del Raspeig, s/n Sant Vicent del Raspeig. Código postal: 03690. 


\title{
RESUMO
}

Este artigo se insere na linha de reflexão em educação matemática centrada em caracterizar e desenvolver a competência docente "olhar profissionalmente" no ensino-aprendizagem das matemáticas como um componente da prática profissional do professor de matemática. Este exemplo procedente de um curso de formação inicial de professores permite caracterizar seu significado. No artigo argumenta-se que o desenvolvimento dessa competência docente apoia-se na relação dialética entre o conhecimento da matemática para o ensino (MKT) e o discurso gerado na resolução de tarefas profissionais. Finalmente descrevem-se algumas características dos ambientes de aprendizagem nos programas de formação de professores dirigidos a fomentar o desenvolvimento dessa relação dialética.

Palavras-chave: olhar profissionalmente; formação inicial de professores; conhecimento de matemática para o ensino; prática profissional do professor de matemática.

\begin{abstract}
This report provides some reflections on the "professional noticing" skill as a component of professional practice of the mathematics teacher. We provide an example from a pre-service mathematics teachers program in order to characterize the aforementioned skill. We claim that the development of professional noticing is supported by the dialectic relation between mathematics knowledge for teaching (MKT) and the discourse generated in the resolution of professional tasks. Finally, some aspects of the learning environments in teacher education programs are described in order to support the relation between MKT and the resolution of professional tasks.

Keywords: professional noticing; pre-service teacher education; mathematical knowledge for teaching; mathematics teacher professional practice.
\end{abstract}

\section{Introducción}

Este artículo se inserta en la línea de reflexión en educación matemática centrada en caracterizar y desarrollar la competencia docente "mirar profesionalmente" la enseñanza-aprendizaje de las matemáticas como una componente de la práctica profesional del profesor de matemáticas (LLINARES, 2012; LLINARES; VALLS, 2009, 2010; JACOBS; LAMB; PHILIPP, 2010; MORRIS; HIEBERT; SPITZER, 2009; SHERIN; JACOBS; PHILIPP, 2010). Esta idea se vincula a la 
necesidad de preparar a los estudiantes para profesor para que aprendan desde la práctica (HIEBERT; MORRIS; BERT; JANSEN, 2007; LLINARES; KRAINER, 2006) lo que implica aprender a analizar diferentes aspectos de la enseñanza (las tareas matemáticas, el aprendizaje del estudiante producto de la resolución de las tareas matemáticas, la interacción y el discurso matemático en el aula).

La competencia docente "mirar profesionalmente" como una componente de la práctica profesional del profesor de matemáticas permite al profesor de matemáticas ver las situaciones de enseñanza-aprendizaje de una manera que lo diferencia de la manera de mirar de alguien que no es profesor de matemáticas. Aunque en los últimos años esta competencia ha sido conceptualizada desde diferentes perspectivas, la idea común en estos planteamientos es subrayar la manera en la que los profesores interpretan las situaciones de enseñanza de las matemáticas. Mason (2002) indica como una característica de esta competencia docente el que el profesor sea consciente de cómo interpreta las situaciones de enseñanza-aprendizaje, mirando de una manera estructurada lo que puede ser relevante. Una característica de esta perspectiva interpretativa es la relación con el conocimiento de matemáticas del profesor, es decir, la interdependencia de la comprensión matemática del profesor con la competencia "mirar profesionalmente" (FERNÁNDEZ; LLINARES; VALLS, 2011, 2012, 2013; PREDIGER, 2010; BARTELL; WEBEL; BOWEN; DYSON, 2013). De esta manera, el análisis de la enseñanza de las matemáticas está relacionado con el aprendizaje a lo largo de la vida del profesor.

El significado de esta competencia docente está vinculado a los intentos de comprender la manera en la que el profesor "usa el conocimiento de matemáticas para la enseñanza" cuando realiza diferentes tareas profesionales (Figura 1) (FORTUNY; RODRIGUEZ, 2012; PONTE; QUARESMA; BRANCO, 2012). En este contexto, las reflexiones sobre la idea del "conocimiento de matemáticas para la enseñanza" (BALL; THAMES; PHELPS, 2008) subrayan la necesidad de considerar la especificidad del conocimiento necesario para realizar el análisis de la enseñanza de las matemáticas. Esta manera de relacionar las destrezas vinculadas al análisis de la práctica (la competencia docente) con la especificidad del conocimiento de matemáticas para la enseñanza se apoya en la necesidad de potenciar el aprendizaje desde la práctica a lo largo de la vida profesional del profesor.

En este caso, la identificación de las tareas que organizan la práctica profesional del profesor de matemáticas (Figura 1) es relevante por dos cosas. Primero, por la identificación de los contextos en los que el profesor usa el conocimiento y por tanto en la identificación de las tareas en las que debe ser competente y, segundo, por el reconocimiento de la influencia del contexto en la manera en la que se usa el conocimiento. Esta idea pone de relieve la relación dialéctica entre el conocimiento teórico y la práctica y el desarrollo de esta com- 


\section{Sistemas de actividad en la enseñanza de las matemáticas como una práctica}

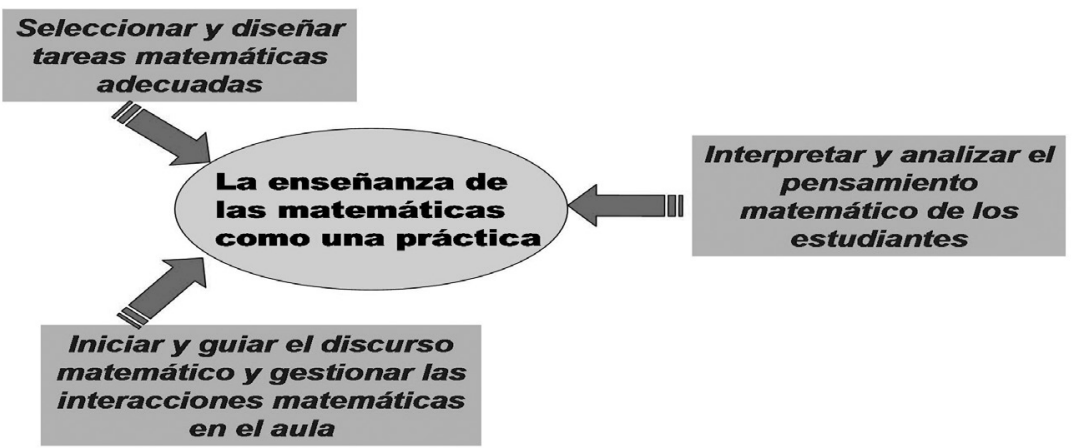

FIGURA 1 - SISTEMA DE ACTIVIDAD EN LA PRÁCTICA PROFESIONAL DE PROFESOR DE MATEMÁTICAS

petencia docente. Es, en este sentido, en el que la relación entre el conocimiento del profesor y la práctica profesional genera la idea del "uso del conocimiento en contexto" que deriva en la noción de competencia docente del profesor de matemáticas. De esta manera el "uso del conocimiento en la práctica" (la competencia docente) se convierte en un foco relevante para comprender mejor la práctica profesional del profesor y su conocimiento profesional (conocimiento de matemáticas para enseñar).

Por otra parte, la competencia docente mirar profesionalmente la enseñanza-aprendizaje de las matemáticas se apoya en el desarrollo por parte del profesor de una perspectiva interpretativa de la enseñanza de las matemáticas mediante el uso en contexto del conocimiento de matemáticas para enseñar (BALL; THAMES; PHELPS, 2008; HILL et al., 2008; SHERIN, 2001; SHERIN; JACOBS; PHILIPP, 2010). La importancia de esta competencia docente emerge al asumir que los estudiantes para profesor y los profesores necesitan seguir aprendiendo a lo largo de su vida profesional y que la "expertise" se va consiguiendo paulatinamente. La destreza que puede permitir al profesor aprender desde la práctica a lo largo de su vida profesional es la competencia docente mirar de manera profesional.

Estas reflexiones plantean un desafío a los formadores de profesores de matemáticas ya que deben diseñar contextos en los programas de formación (materiales-tareas y aproximaciones metodológicas) para que se inicie el desarrollo de esta competencia de manera que permita a los estudiantes para profesor aprender a aprender a lo largo de su vida profesional. 


\section{Analizar tareas matemáticas como contexto para el desarrollo de la competencia docente}

El siguiente ejemplo procede de un curso de formación inicial de profesores y nos permite caracterizar diferentes grados de desarrollo de lo que significa mirar profesionalmente (PRIETO; VALLS, 2010; SANCHEZ-MATOMOROS; FERNÁNDEZ; VALLS; GARCÍA; LLINARES, 2012). Estos ejemplos muestran evidencias de los desafíos a los que se enfrentan los estudiantes para profesor cuando tienen que usar el conocimiento de matemáticas específico para la enseñanza en el análisis de tareas matemáticas y muestran que la competencia docente "mirar profesionalmente" no es innata y que debe empezar a desarrollarse en los programas de formación de profesores.

La actividad que nos sirve para ejemplificar estas características estaba centrada en apoyar a los estudiantes para profesor a analizar tareas matemáticas que pueden ser usadas en una lección. La elección y adaptación de las tareas matemáticas es una actividad profesional importante del profesor así como la gestión que hace durante la enseñanza (PENALVA; LLINARES, 2011). El análisis del profesor de las tareas matemáticas para planificar una lección para sus alumnos moviliza su conocimiento de matemáticas en relación al aprendizaje de los alumnos. La manera en la que el estudiante para profesor identifica lo relevante de la tarea matemática y cómo lo vincula el aprendizaje pretendido con sus alumnos a los objetivos curriculares es una manifestación de la competencia docente (MORRIS et al., 2009). Es decir, la competencia docente para analizar tareas matemáticas viene determinada por la manera en la que el estudiante para profesor identifica la actividad matemática que la tarea puede potenciar en sus alumnos (la idea de demanda cognitiva de la tarea matemática). La Figura 2 muestra una de las tareas de análisis propuestas en la que se pedía a los estudiantes para profesor que describieran y analizaran las características de las tareas matemáticas que aparecían en una Web de recursos relativo al tema de los cuadriláteros para alumnos de 12-13 años. La Figura 3 muestra una de las tareas que aparecían en esta Web. La característica de las tareas matemáticas en esta Web es que presenta a los alumnos actividades matemáticas diseñadas con applets que permiten modificar los parámetros que definen polígonos para generar cuadriláteros de diferentes características. 
Tarea. Analizad las tareas en la unidad didáctica desde Descartes 2.0 (Matemáticas interactivas, Ministerio de Educación, Cultura y Deporte)

Nivel. $1^{\circ}$ Educación Secundaria Obligatoria (12-13 años)

Tema: Los cuadriláteros (9 escenas)

Tema: Ángulos en polígonos regulares (10 escenas)

http://recursostic.educacion.es/descartes/web/indice ud.php

1. Identificad la demanda cognitiva de cada una de las tareas propuestas

¿Qué ideas matemáticas se desarrollan en cada actividad?

Relacionad cada una de las características generales de los niveles de demanda cognitiva que aparecen en el documento de apoyo con las ideas que desarrolla cada actividad.

2. Estableced un cuadro resumen indicando el número de actividades en cada nivel de demanda cognitiva en cada Unidad Didáctica (UD).

3. Estableced la relación entre las actividades, o grupos de ellas, y los objetivos del currículo.

4. Para la UD de los Cuadriláteros: Compara el tipo de actividades en cada una de estas UD de Descartes 2.0 y las actividades propuestas en

http://mathforum.org/ annie/gsp.handouts/allquad/

según el diferente nivel de demanda cognitiva de las tareas en la UD y las investigaciones propuestas en mathforum.

FIGURA 2 - TAREA PROFESIONAL EN EL PROGRAMA DE FORMACIÓN DE PROFESORES CENTRADA EN EL DESARROLLO DE LA COMPETENCIA DOCENTE VINCULADAAL ANÁLISIS DE TAREAS MATEMÁTICAS ("MIRAR CON SENTIDO LAS TAREAS MATEMÁTICAS”)

Los estudiantes para profesor debían analizar las diferentes tareas que aparecían en la Web considerando su demanda cognitiva. La información teórica que podía ayudar a los estudiantes para profesor a realizar este análisis se les proporcionaba en un documento de texto que resumía la información descrita en Penalva \& Llinares (2011). En esta actividad centrada en los paralelogramos los elementos matemáticos a considerar eran:

- las definiciones de los diferentes cuadriláteros;

- las relaciones de inclusión entre los diferentes tipos de cuadriláteros;

- las relaciones entre ángulos, lados y diagonales en los paralelogramos.

Los diferentes niveles de demanda cognitiva que debían ser considerados en el análisis de las tareas eran: 
FIGURA 3 - UNA DE LAS TAREAS SOBRE CUADRILÁTEROS QUE DEBÍAN SER ANALIZADAS POR LOS ESTUDIANTES PARA PROFESOR CONSIDERANDO LA DEMANDA COGNITIVA PARA EL RESOLUTOR. Disponível em: < http://recursostic.educacion.es/descartes/web/ materiales_didacticos/los_cuadrilateros/cuadrilateros1.htm> 
- Nivel 1- tareas de memorización;

- Nivel 2- tareas de procedimientos sin conexión;

- Nivel 3- tareas de procedimientos con conexión;

- Nivel 4- tareas que requieren "hacer matemáticas".

La manera en la que los estudiantes para profesor usaban los diferentes elementos matemáticos y las características de la demanda cognitiva para realizar el análisis pone de manifiesto diferentes grados de desarrollo de la competencia docente "mirar profesionalmente". Es decir, la manera en la que los estudiantes para profesor analizan las tareas matemáticas son ejemplos del uso del conocimiento matemático para la enseñanza en la realización de una tarea profesional. En este sentido, la manera en la que los estudiantes para profesor "veían" las tareas matemáticas determina en qué medida consideran que estas tareas proporcionan oportunidades efectivas para aprender un contenido matemático relevante para sus alumnos (MORRIS et al., 2009). Los estudiantes para profesor tenían que responder a las cuestiones siguientes:

Las respuestas producidas muestran de que manera los estudiantes para profesor estaban "viendo" el potencial de estas tareas matemáticas para el aprendizaje de sus alumnos y qué variables de la tarea identificaban como potencialmente modificables para adaptar las tareas matemáticas a algún grupo de alumnos particulares.

\section{Cuestión 1}

- Identificad la demanda cognitiva de cada una de las tareas propuestas.

- ¿Qué ideas matemáticas se desarrollan en cada actividad?

- Relacionad cada una de las características generales de los niveles de demanda cognitiva que aparecen en el documento de apoyo con las ideas que desarrolla cada actividad.

\section{Cuestión 3}

- Estableced la relación entre las actividades, o grupos de ellas, y los objetivos del currículo. 
Estudiante para profesor A

\section{ESCENA 2 - LOS PARALELOGRAMOS}

Cuestión 1

En esta escena, la actividad está muy dirigida. El alumno solo tiene que seguir las instrucciones, arrastrando vértices, anotando datos, comprobando que se cumplen las propiedades de los paralelogramos. Tienen que comprobar el teorema de Pitágoras porque claramente se le exige al alumno en el enunciado. Por tanto se requiere un nivel 1 de demanda cognitiva ya que solo tienen que reproducir las fórmulas y definiciones de las propiedades de los paralelogramos.

Los últimos apartados de esta escena requieren un nivel de demanda cognitiva de nivel 2 ya que requieren una explicación, pero más que desarrollar una comprensión matemática buscan producir respuestas correctas. El hecho de aplicar el Teorema de Pitágoras requiere entender la descomposición del rectángulo y del rombo y relacionar conceptos, por tanto aquí ya se está exigiendo un esfuerzo cognitivo que requiere un nivel 3 de demanda cognitiva. La idea matemática que se desarrolla es la construcción de paralelogramos conociendo algunos datos y aplicando sus propiedades. Se desarrolla la capacidad de descomposición de estos paralelogramos y la capacidad de extracción visual de los triángulos que componen estos, ayudando al alumno a comprender los conceptos, ya que aplican el Teorema de Pitágoras para obtener un dato del rectángulo.

\section{Cuestión 3}

Tanto en aquellas tareas que exigen comentar lo observado tras realizar el ejercicio como las que requieren un mayor esfuerzo cognitivo relacionando conceptos e ideas matemáticas, lo que se pretende es que el alumno mejore la capacidad de pensamiento reflexivo e incorpore al lenguaje y modos de argumentación las formas de expresión y razonamiento matemático con el fin de comunicarse de manera, clara, precisa y concisa. Este objetivo coincide con el primero que encontramos en el currículo de la Educación Secundaria Obligatoria (ESO).

En aquellas tareas que únicamente exigen recordar y aplicar fórmulas, se está buscando que el alumno aplique con soltura las herramientas matemáticas, aunque las propuestas en Descartes no se aplican a ninguna situación de la vida cotidiana, como incluye el objetivo número 2 del currículo de la ESO. Sin embargo, todas las tareas propuestas en Descartes buscan utilizar de forma adecuada este applet, exigiendo al alumno que experimente con él, arrastrando 
vértices y construyendo nuevas figuras, al igual que dice el currículo en su objetivo número 7.

En mi opinión, las tareas en las que se exige al alumno dibujar una figura diferente a la de la escena pueden hacer que el alumno desarrolle una actitud positiva, sintiéndose capaz de hacerlo y así reforzar su autoestima. El currículo de la ESO también tiene un objetivo parecido y dice así: Manifestar una actitud positiva muy preferible a la actitud negativa ante la resolución de problemas y mostrar confianza en la propia capacidad para enfrentarse a ellos con éxito y adquirir un nivel de autoestima adecuado, que les permita disfrutar de los aspectos creativos, manipulativos, estéticos y utilitarios de las Matemáticas.

Este estudiante para profesor identifica dos demandas cognitivas para los estudiantes entre las cuestiones que solo implican construir la figura y realizar medidas (por ejemplo la cuestión f) (Un cuadrado de lado 12 unidades de longitud. Comprueba la medida de las diagonales) y las que implican realizar alguna conjetura y comprobarla empíricamente que conllevan la identificación de las propiedades de los paralelogramos en relación a la igualdad de las diagonales, la igualdad de los ángulos opuestos y la relación entre la longitud de los lados del paralelogramo y los ángulos que forman las diagonales (cuando las diagonales son iguales, los cuatro ángulos en el paralelogramo son iguales. ¿Qué relación hay entre las longitudes de las diagonales de los paralelogramos de cada clase?, y ¿Observas alguna característica en el ángulo que forman las diagonales en cada clase de paralelogramo?).

La identificación por parte del estudiante para profesor de los elementos matemáticos que caracterizan la tarea matemática y la demanda cognitiva que supone (diferencia entre realizar medidas y realizar conjeturas) en cada una de las tareas que aparecen en la lección son evidencias de la competencia docente. Por ejemplo cuando el estudiante para profesor A indica "El hecho de aplicar el Teorema de Pitágoras requiere entender la descomposición del rectángulo y del rombo y relacionar conceptos, por tanto aqui ya se está exigiendo un esfuerzo cognitivo que requiere un nivel 3 de demanda cognitiva".

Sin embargo, algunos estudiantes para profesor tienen mayor dificultad en identificar los elementos matemáticos en las tareas que son relevantes para el aprendizaje de los alumnos. Estos estudiantes para profesor suelen referirse de manera general a las tareas y consideran las secuencias de manera global. Por ejemplo, lo que sigue es la respuesta de otro estudiante para profesor a la misma tarea anterior (Figura 2). 
Estudiante para profesor B

\section{Cuestión 1}

En el Tema 1, Los Cuadriláteros, nos encontramos ante una demanda cognitiva de nivel bajo, concretamente de Nivel 1, al intentar cumplir los objetivos de reconocimiento de cualquier tipo de cuadrilátero y la resolución de su perímetro y su área. Se trata pues de reproducir fórmulas que no son ambiguas, ya vistas en clase anteriormente y que no ponen evidencia a lo estudiado en clase.

Sin embargo, si nos fijamos en cada actividad de diferente tipo de cuadrilátero: paralelogramos, trapecios, cometas, podemos ver que existen actividades un poco más complejas, de Niveles 2 y 3 , en las que se exigen algunas reflexiones más profundas a los estudiantes. Esto es debido a que se pide buscar relaciones entre los lados y ángulos de las figuras, así como el dibujo de nuevas figuras de otras mediciones para después estudiar, encontrar características y relaciones entre ellas y relacionar con otros conceptos como el Teorema de Pitágoras.

\section{Cuestión 3}

- Todas las actividades, por su carácter de ser realizadas Online, intentan utilizar de forma adecuada los distintos medios tecnológicos, en este caso Internet. También intentan integrar los conocimientos matemáticos en el conjunto de saberes que se van adquiriendo desde las distintas materias de modo que puedan emplearse de forma creativa, analítica y crítica.

- Las actividades procedentes de ambos temas, Los Cuadriláteros y los Ángulos en Polígonos Regulares, tratan de mejorar la capacidad de pensamiento reflexivo, así como identificar las formas planas o espaciales que se presentan en la vida diaria y analizar las propiedades y relaciones geométricas entre ellas

En su respuesta el estudiante para profesor B reproduce los elementos matemáticos que aparecen en el enunciado de las tareas matemáticas (cuadrilátero, perímetro, área, teorema de Pitágoras) pero se refiere de manera global a todas las tareas y menciona la "demanda cognitiva" indicando "actividades un poco más complejas" aunque indica que la actividad demanda al alumno ("buscar relaciones entre los lados y ángulos de las figuras") sin indicar a qué relación se refiere en cada tarea específica. La generalidad con la que este estudiante para profesor responde en relación al análisis de las tareas matemáticas le impide 
relacionar los elementos matemáticos de las tareas (y la demanda cognitiva) con los objetivos de aprendizaje pretendido (objetivos del currículo).

El desafío para los estudiantes para profesor que plantean las tareas de análisis de las lecciones desde la perspectiva del aprendizaje matemático pretendido en los alumnos muestra la complejidad de la competencia docente que implica usar el conocimiento de las matemáticas para la enseñanza. En este sentido, el uso del conocimiento de matemática especializado para la enseñanza que se moviliza en la realización de esta tarea profesional es una manifestación del nivel de competencia docente y los ejemplos anteriores indican que el uso de la información teórica no se realiza de manera espontánea. Por lo tanto, el hecho de que el desarrollo de esta competencia docente no sea un espontáneo plantea la necesidad de articular en los programas de formación de profesores oportunidades para apoyar su desarrollo.

\section{El desarrollo de la competencia docente "mirada profesional" del profesor de matemáticas}

Una característica de los entornos de aprendizaje diseñados en los programas de formación de profesores para fomentar el desarrollo de la competencia docente "mirar profesionalmente" la enseñanza-aprendizaje de las matemáticas es que se asume la relación dialéctica entre la construcción del conocimiento y el discurso (BALL; SLEEP; BOERST; BASS, 2009; BARTELL; WEBEL; BOWEN; DYSON, 2013; WELLS, 2002). Esta hipótesis procede de considerar perspectivas socioculturales en el diseño de los entornos de aprendizaje. Algunas de las investigaciones realizadas considerando la relación entre la generación de un discurso argumentativo por parte de los estudiantes para profesor durante la resolución de tareas profesionales y la construcción del conocimiento aportan evidencias del papel que desempeña la interacción en la discusión guiada por un objetivo en los programas de formación de profesores (LLINARES, 2012; ROIG; LLINARES; PENALVA, 2011; PENALVA; REY; LLINARES, 2011).

La adopción de perspectivas socioculturales para comprender el desarrollo de la competencia docente "mirada profesional" en los programas de formación de profesores implica considerar que los profesores/estudiantes para profesor tienen diferentes oportunidades para dotar de sentido a la práctica de enseñar matemáticas. En estas oportunidades de aprendizaje hay que tener en cuenta diferentes aspectos: el conocimiento previo (la experiencia), el acceso a la información teórica, contextos en los que la interacción y el discurso favorecen los 
procesos de construcción del conocimiento, y finalmente la nueva comprensión conseguida de las situaciones de enseñanza-aprendizaje (LLINARES, 2012). Estos aspectos del desarrollo de la competencia docente "mirar profesionalmente" se pueden concretar en las siguientes actividades/tareas en los entornos de aprendizaje (Figura 4):

- Usar registros de la práctica: video-clips de lecciones de matemáticas, respuestas de alumnos a problemas, secuencia de actividades/tareas en una lección, ...;

- Leer documentos con información teórica relativa a la tarea de análisis de los registros de la práctica. Estos documentos con información teórica proporcionan a los profesores/estudiantes para profesor referentes para identificar los aspectos matemáticos relevantes y los dotan de instrumentos para su interpretación;

- Usar contextos de interacción: Contextos en los que la interacción entre los profesores permite organizar, explicitar, refinar y justificar las interpretaciones de los registros de la práctica que a su vez permiten explicitar el conocimiento previo y a veces implícito que se tiene sobre la práctica. Estos contextos de interacción pueden ser debates en-línea o discusiones en gran grupo y/o pequeño grupo de manera presencial;

- Sintetizar las interpretaciones de los registros de la práctica y sus justificaciones en documentos (reportes) en los que la nueva comprensión de los registros de la práctica es sistematizada y organizada.

En este sentido, las reflexiones aportadas durante el CIAEM (CONFERENCIA INTERAMERICANA DE EDUCACIÓN MATEMÁTICA, Recife, Brasil, 2011) en relación al desarrollo de la competencia docente "mirar profesionalmente" la enseñanza de las matemáticas y el diseño de entornos de aprendizaje en un programa de formación (LLINARES, 2011) subrayaban que:

- el "conocimiento en uso" que configura la competencia docente "mirar profesionalmente" se ve como el uso de instrumentos tanto físicos como conceptuales en la identificación e interpretación de aspectos relevantes en la enseñanza-aprendizaje de las matemáticas;

- el desarrollo de la competencia docente "mirar profesionalmente" se ve como la transformación de la persona mediante la participación creciente en "prácticas sociales" en función de la naturaleza de las tareas y actividades que resuelven.

Esta perspectiva del desarrollo de la competencia docente tiene implicaciones en el diseño de los materiales de enseñanza para el programa de formación 


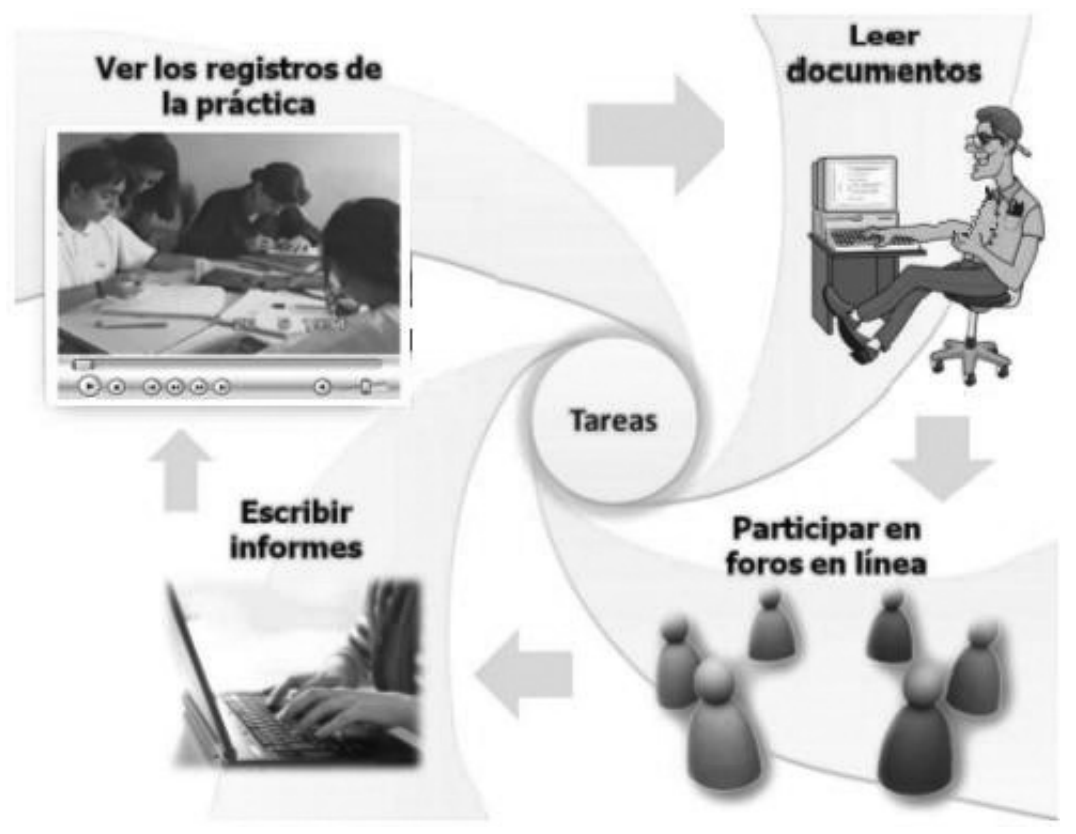

FIGURA 4 - ACTIVIDADES Y RELACIONES EN ENTORNOS DE APRENDIZAJE

y los espacios de interacción que tienen como objetivo el que los estudiantes para profesor construyan conocimiento necesario para enseñar matemáticas (BALL et al., 2008; FERNÁNDEZ; LLINARES; VALLS, 2013; HILL et al., 2008; PREDIGER, 2010) y plantea todavía muchos interrogantes sobre las decisiones que hay que tomar al pensar en cómo diseñar las oportunidades para aprender. Sin embargo, al ser la actividad de formar profesores una actividad institucionalizada hace que no sea fácil tomar decisiones únicamente considerando lo que los análisis teóricos nos dicen. Lo descrito en este articulo intenta subrayar que la competencia docente "mirar profesionalmente" del profesor de matemáticas considerada como una componente de la práctica profesional del profesor de matemáticas no se debe reducir a poseer una serie de conocimientos. El énfasis está situado en el uso del conocimiento en la realización de tareas interpretativas de los registros de la práctica como una manera de empezar a desarrollar el proceso de aprender de la práctica. En este sentido, el "uso del conocimiento" guiado por un objetivo es lo que nos permite usar la palabra "profesional" junto con la idea de competencia docente "mirada profesional" del profesor de matemáticas. 


\section{REFERENCIAS}

BALL, D. L.; SLEEP, L.; BOERST, T.; BASS, H. Combining the development of practice and the practice of development in teacher education, 2009.

BALL, D. L.; THAMES, M. H.; PHELPS, G. Content knowledge for teaching: What makes it special?. Journal of Teacher Education, n. 59, p. 389-407, 2008.

BARTELL, T. G.; WEBEL, C.; BOWEN, B.; DYSON, N. Prospective teacher learning: recognizing evidence of conceptual understanding. Journal of Mathematics Teacher Education, n. 16, p. 57-79, 2013.

FERNÁNDEZ, C.; LLINARES, S.; VALLS, J. Learning to notice students' mathematical thinking through online discussions. ZDM. Mathematics Education, n. 44, p. 747-759, 2012 .

. "Mirando con sentido" el pensamiento Matemático de los estudiantes sobre la razón y proporción. Acta Scientiae, Canoas, Brasil, v. 13, n. 1, p. 9-30, jan./jun. 2011.

. Primary Teacher's Professional Noticing of Students' Mathematical Thinking. The Mathematics Enthusiast. Special Issue: International Perspectives on Problem Solving Research in Mathematics Education, v. 10, n. 1\&2 (Information Age Publishing), 2013.

FORTUNY, J. M.; RODRÍGUEZ, R. Aprender a mirar con sentido: facilitar la interpretación de las interacciones en el aula. AIEM. Avances de Investigación en Educación Matemática, n. 1, p. 23-37, 2012.

HIEBERT, J.; MORRIS, A. K.; BERT, D.; JANSEN, A. Preparing teachers to learn from teaching. Journal of Teacher Education, n. 58, p. 47-61, 2007.

HILL, H. C.; BLUNK, M. L.; CHARALAMBOUS, CH. Y.; LEWIS, J. M.; PHELPS, G. C.; SLEEP, L.; BALL, D. L. Mathematical Knowledge for Teaching and the mathematical Quality of Instruction: An Exploratory Study. Cognition and Instruction, n. 26, p. 430-511, 2008.

JACOBS, V. R.; LAMB, L. C.; PHILIPP, R. Professional noticing of children's mathematical thinking. Journal for Research in Mathematics Education, v. 41, n. 2, p. 169 202, 2010.

LLINARES, S. Construcción de conocimiento y desarrollo de una mirada profesional para la práctica de enseñanza matemáticas en entornos en línea. AIEM. Avances de Investigación en educación Matemática, n. 2, p. 53-70, 2012.

. Formación de profesores de matemáticas. Caracteriziación y desarrollo de competencias docentes. In: CIAEM - CONFERENCIA INTERAMERICANA DE EDUCACIÓN MATEMÁTICA, XIII., 2011, Recife, Brasil. Anais... Recife, Brasil, Junio, 2011.

LLINARES, S.; KRAINER, K. Mathematics (student) teachers and teacher educators as learners. In: GUTIERREZ, A.; BOERO, P. (Eds.). Handbook of Research on the 
Psychology of mathematics Education: Past, Present and Future. Sense Publishers: Rotterdam/Taipei, 2006. p. 429-459.

LLINARES, S.; VALLS, J. Prospective primary mathematics teachers' learning from online discussions in a virtual video-based environment. Journal of Mathematics Teacher Education, v. 13, n. 2, p. 177-196, 2010.

The building of pre-service primary teachers' knowledge of mathematics teaching: interaction and online video case studies. Instructional Science, v. 37, n. 2, p. 247-271, 2009.

MASON, J. Researching your own practice. The discipline of noticing. Routledge Falmer: Londres, 2002.

MORRIS, A. K.; HIEBERT, J.; SPITZER, S. Mathematical Knowledge for Teaching in Planning and Evaluating Instruction: What Can Preservice Teachers Learn?. Journal for Research in Mathematics Education, v. 40, n. 5, p. 491-529, 2009.

PENALVA, M. C.; LLINARES, S. Tareas matemáticas en la educación secundaria. En: GOÑI, J. M. (Ed.). Didáctica de las matemáticas. GRAO: Barcelona, 2011. p. 27-74.

PENALVA, M. C.; REY, C.; LLINARES, S. Identidad y aprendizaje de estudiantes de psicopedagogía. Análisis en un contexto b-learning en didáctica de la matemática. Revista Española de Pedagogía, n. 248, p. 101-118, 2011.

PONTE, J. P.; CHAPMAN, O. Mathematics Teachers' Knowledge and Practices. En: GUTIERREZ, A.; BOERO, P. (Eds.). Handbook of Research on the Psychology of mathematics Education. Past, Present and Future. Sense Publishers: Rotterdam/Taipei, 2006. p. 461-494.

PONTE, J. P.; QUARESMA, M.; BRANCO, N. Práticas profissionais dos professores de Matemática. AIEM. Avances en Investigación en Educación Matemática, n. 1, p. 65-86, 2012.

PREDIGER, S. How to develop mathematics-for-teaching and for understanding: the case of meanings of the equal sign. Journal of Mathematics Teacher Education, n. 13, p. 73-93, 2010.

PRIETO, J. L.; VALLS, J. Aprendizaje de las características de los problemas aritméticos elementales de estructura aditiva en estudiantes para maestro. Educación Matemática, v. 22, n. 1, p. 57-85, 2010.

ROIG, A. I.; LLINARES, S.; PENALVA, M. C. Estructuras argumentativas de estudiantes para profesores en un entorno en línea. Educación Matemática, v. 23, n. 3, p. 39-65, 2011.

SÁNCHEZ-MATAMOROS, G.; FERNÁNDEZ, C.; VALLS, J.; GARCIA, M.; LLINARES, S. Cómo estudiantes para profesor interpretan el pensamiento matemático de los estudiantes de Bachillerato. La derivada de una función en un punto. En: ESTEPA, A.; CONTRERAS, Á.; DEULOFEU, J.; PENALVA, M. C.; GARCÍA, F. J.; ORDÓÑEZ, L. (Eds.). Investigación en Educación Matemática XVI. Jaén: SEIEM, 2012. p. 497-508. 
SHERIN, M. G. Developing a professional vision of classroom events. In: WOOD, T.; NELSON, B. S.; WARFIELD, J. (Eds.). Beyond classical pedagogy: Teaching elementary school mathematics. Mahwah, NJ: Erlbaum, 2001.p. 75-93.

SHERIN, M. G.; JACOBS, V. R.; PHILIPP, R. A. (Eds.). Mathematics teacher noticing: Seeing through teachers' eyes. New York: Routledge, 2010.

WELLS, G. Dialogic inquiry. Towards a sociocultural practice and theory of education. 2. ed. Cambridge: Cambridge University Press, 2002.

Texto recebido em 23 de julho de 2013.

Texto aprovado em 12 de agosto de 2013. 